\title{
I Zum Verhältnis von Texten, Bildern und Illustrationen in der Konstruktion von Subjektpositionen am Beispiel des (bewusst) Konsumierenden
}

\author{
Michael Brandmayr
}

\section{Zusammenfassung}

Dieser Beitrag stellt anhand eines Beispiels dar, wie die Subjektpositionen des „bewussten Konsumierenden“ in Grafiken und Bildern konstituiert und dem Betrachtenden vermittelt werden. Dazu wird das Bild Your Own Personal Slaves von Daniel Garcia mit der Drei-Schritt-Methode nach Panofsky analysiert. Der ikonologische Gehalt des Bildes wird im Hauptteil des Textes interpretiert. Die These lautet dabei, dass auch in der gegenwärtigen Akzentuierung von bewussten Konsumpraktiken die bereits bei Veblen bemerkte Funktion des Konsums, Disktinktion herzustellen, nachgewiesen werden kann. Nicht nur entspricht die Fokussierung einer nachhaltigen, bewussten Konsumweise stärker der Konsumpraxis mittlerer und oberer Schichten und ihren finanziellen Möglichkeiten; die Positionierung dieser Praxis als moralisch höher stehend und objektiv vernünftig liefert Gründe, ein abweichendes Verhalten als rückständig zu klassifizieren und abzuwerten. Anhand dieses Beispiels sollen im letzten Teil des Artikels zwei Thesen diskutiert werden, die argumentieren, dass Bilder gegenüber Texten über eine bestimmte Einzigartigkeit bei der Konstruktion von Subjektivität verfügen.

Schlagwörter: Subjektivierung, Bildanalyse, Klassismus, Konsum

On the relationship of texts, images and illustrations in the construction of subject positions using the example of the (conscious) consumer

Abstract

This contribution uses an example to show how the subject positions of the "conscious consumer" are constituted in graphics and images and conveyed to the viewer. For this purpose, the image "your personal slaves" by Daniel Garcia will be analyzed with the three step method according to Panofsky. The iconological content of the image is interpreted in the main part of the text. The thesis here is that even in the current accentuation of conscious consumption practices, the function of consumption, already noticed in Veblen's work, namely to produce discourse, can be demonstrated. Not only does the focus on a sustainable, conscious mode of consumption correspond more closely to the consumption practice of middle and upper classes and their financial possibilities; the positioning of this practice as morally superior and objectively reasonable provides reasons to classify deviant behaviour as backward and to devalue it. Based on this example, the last part of the article will discuss two theses that argue that images have a certain uniqueness in the construction of subjectivity compared to texts.

Keywords: subjectification, image analysis, classism, consumption

IIIIIIIIIIIIIIIIIIIIIIIIIIIIIIIIIIII

${ }^{*}$ Michael Brandmayr, Ph.D., ist Sozialarbeiter der Kinder- und Jugendhilfe der Stadt Innsbruck und lehrt nebenberuflich an den Universitäten Innsbruck, Wien und Linz in den Bereichen Bildungssoziologie und politische Bildung. E-Mail: michael.brandmayr@uibk.ac.at

2020 | innsbruck university press | Innsbruck

Momentum Quarterly I ISSN 2226-5538 I momentum-quarterly.org Vol. 9, No 3 I DOI 10.15203/momentumquarterly.vol9.no3.p151-160 


\section{Einleitung}

In den vergangenen Jahren hat sich innerhalb der Diskursanalyse und Subjektivierungsforschung ein stärkeres Interesse an symbolischen und bildlichen Elementen in der Wissenschaft gezeigt (Traue 2013; Kauppert/Leser 2014; Geimer/Burghardt 2019). Es scheint klar, dass Bilder, Grafiken, Videos, GIFs und andere Formate nicht nur auf einen bestimmten Topos oder ein Narrativ Bezug nehmen, sondern dass sie dessen Gegenständlichkeit im Diskurs mitkonstituieren. Sie haben im Prozess der Anrufung (Althusser 1971; Butler 2001) und der Subjektkonstitution weitreichendere Effekte, indem sie an emotionale, symbolische und imaginäre Dimensionen appellieren können. Allein bildhafte Elemente sind an vielen Stellen in der Lage auszudrücken, was Textliches nicht vermag, und sind so essenzieller Baustein in der Produktion diskursiver Gegenstände. Doch wie diese Ko-konstruktion vonstatten geht und wie das Zusammenspiel von textbasierten und bildhaften Diskurselementen funktioniert, ist bisher noch wenig erforscht.

Ich möchte in diesem Beitrag zumindest einige Hinweise geben, wie dies denkbar ist. Dabei verfolge ich die Frage des Konsums, genauer: der Veränderungen des Deutungsmusters von Konsum und damit der Subjektposition des/der Konsument/in, und untersuche diese Deutungsmuster in einer Kombination von Diskurs- und Bildanalyse. Ich werde (ohne auf die entsprechende umfassende theoretische Diskussion Bezug nehmen zu können) versuchen, diese Wechselwirkung an einem Bild sowie an ausgewählten textlichen Diskurselementen zur Frage des bewussten Konsums darzustellen. Die These lautet dabei, dass die Konstruktion der Subjektposition von „abgehängten Konsumierenden“ über die Ausgrenzung ihrer kulturellen Alltagspraktiken vollzogen wird (und weniger in einem objektiven Wandel dieser Praktiken), indem diese Praktiken als gleichzeitig unmoralisch wie objektiv unvernünftig positioniert werden, und sich diese Subjektposition besonders symbolisch-bildhaft konstituiert.

Dieser Artikel verfolgt also zwei Anliegen: Einerseits möchte er einige Thesen über die Wirkung von Bildern im Prozess der Subjektivierung sowie eine Möglichkeit deren Analyse präsentieren; andererseits die Frage aufgreifen, welche Deutungen im Diskurs um bewussten Konsum prozessiert und (oft hintergründig) vermittelt werden. Um beides gleichzeitig zu unternehmen, wird ein Beispiel - eine Darstellung aus dem Dis- kurs um bewussten Konsum - analysiert, anschließend werden darauf aufbauend Thesen vorgestellt. Indem mit einem Beispiel gearbeitet wird, zeigt sich jedoch die Limitation der Aussagekraft dieses Artikels: Er ist keine vollständige und ausgewogene Untersuchung aller existierenden Deutungen im Diskurs um Konsum und keine Darstellung ihrer Gewichtung; er gibt keine Auskunft über alle vorhandenen Diskurspositionen, sondern fragt nur nach genau einer, ohne festzustellen, wie verbreitet diese ist. Zudem sind auch deutlich mehr Varianten der Text-Bild-Interaktion denkbar als die hier vorgestellte, d. h. der Artikel greift auch die Frage nach dem Verhältnis von Bildern in Prozessen der Subjektivierung keinesfalls umfassend und vollständig auf.

Die Frage des Konsums umfasst mehrere Aspekte: Einerseits die Art und Weise, wie Konsumgüter ausgewählt und als wertvoll erachtet werden, aber auch, welchen Zweck sie im Alltag erfüllen sollen und welche Beziehung Menschen zu den von ihnen gebrauchten Gegenständen herstellen. Soziologische Analysen behaupten übereinstimmend einen Wandel in unserer Konsumkultur. Dabei seien zwei parallele, teils widersprüchliche Trends erkennbar: Einerseits steigt der Güterverbrauch, etwa in der Bekleidungsindustrie, und Menschen in OECD-Ländern erwerben immer mehr Konsumgüter in immer kürzeren Phasen, wobei auch die Industrien mit einem größeren Angebot aufwarten (Bänzinger 2015). Sennett zeigt dabei zudem, dass das Angebot von großen Marken sich im Wesentlichen immer mehr ähnelt („gold-plating“; Sennett 2007: 144). Andererseits zeigt sich ein Trend hin zu „bewussterem Konsum“, bei dem für die Konsumierenden Produkteigenschaften wie lokale, nachhaltige und biologische Produktionsweisen, faire Arbeitsbedingungen, ein schonender Umgang mit der Umwelt und Tieren, die Herstellung aus nachwachsenden Ressourcen, ein möglichst geringer $\mathrm{CO}_{2}$-Ausstoß und anfallender Müll u. a. bedeutsamer werden (Bauman 2013; Johnson/Baumann 2014).

Der ersten der skizzierten Konsumpraktiken, der eine traditionelle Vorstellung der Verwirklichung sozialer Teilhabe über den Konsum und damit dem Versprechen sozialer Marktwirtschaft gemäß dem Slogan „Wohlstand für alle“ entspricht, wird gegenwärtig indes die Subjektform der abgehängten Konsumierenden zugeschrieben, d. h. diese Konsumpraxis wird als rückständig und als Lebensweise unterprivilegierter Schichten gedeutet. Umgekehrt entsteht aus der Praxis des „bewussten Konsums“ bzw. der Subjektform des bewussten Konsumierenden eine Deutung moralisch 
richtigen Handelns und moralischer Höherwertigkeit. Die Vermittlung dieser Subjektformen zeigt sich in Texten und Bildern; für diesen Beitrag möchten ich dies exemplarisch an einer in den sozialen Medien verbreiteten Illustration demonstrieren. Für die Bildanalyse und -interpretation greife ich auf die DreiSchritt-Methode von Erwin Panofsky (1939) zurück.

\section{Analyse}

Das Bild Your Own Personal Slaves ${ }^{1}$ von 2016 stammt vom portugiesischen Künstler Daniel Garcia und wurde in den sozialen Medien mehrere Tausend Mal geteilt, darunter etwa von den Seiten „Occupy Wall Street“ und „Capitalism Kills“, die jeweils mehrere Hunderttausend Follower haben. Garcia versah das Bild bei seiner Veröffentlichung mit dem folgenden Subtext: „Which of these products do you use? I wanted to do a piece about all the products we depend on, that are made or reaped by forced labor, child workers, sex slaves, etc."

Auf der Ebene der vorikonografischen Beschreibung ist im Zentrum des Bildes eine weiße Frau mit ambivalentem Gesichtsausdruck zu erkennen, aus deren rechtem Auge eine Träne fließt. Die Frau ist eher jung, hat kurze blonde Haare, leicht androgyne Gesichtszüge und trägt ein schwarzes T-Shirt mit dem Gesicht des kubanischen Widerstandkämpfers Ernesto Che Guevara, eine rote Hose sowie Flip-Flops mit Blumenmuster. Um sie herum sind andere Menschen gruppiert, die für sie Dienstleistungen verrichten und gerade bei der Arbeit sind; teils sitzt sie auf diesen. Ihr Blick ist nicht auf die Menschen gerichtet, sondern fokussiert geradeaus den Beobachter. Rechts hinter ihr näht eine Frau mit asiatischen Gesichtszügen an ihrem T-Shirt; unter dieser Frau reicht ein schwarzer Mann mit Verfärbungen auf seiner Haut der Frau ein Smartphone; wiederum etwas weiter vorne im Bild sammelt ein Mann mit dunkler Hautfarbe Shrimps, neben ihm liegt eine Tafel Schokolade. Links vorne im Bild arbeitet eine Frau mit leicht dunkler Hautfarbe, wie sie in Ländern Mittelamerikas häufig ist, an der Herstellung von Kosmetika, eine Hanfpflanze ragt hier ins Bild. Dahinter bietet eine hellhäutige junge Frau mit blon-

1 Ausgewählt habe ich dieses Beispiel aufgrund seiner starken Verbreitung und seiner Anschaulichkeit. Es ist Teil einer aktuellen Forschungsarbeit, für die ich mehrere Hundert Bilder, Grafiken und Memes zum Thema Konsum sowie ebenfalls mehrere Hundert Blog- oder Zeitungsartikel $\mathrm{zu}$ diesem Thema aus den vergangenen zehn Jahren analysiere. den Haaren, die fast nackt ist, der Frau im Zentrum einen Laptop mit pornografischen Inhalten an. Ganz hinten rechts im Bild ist ein dunkelhäutiger Mann zu sehen, der in seiner linken Hand einen Sack trägt und in seiner rechten eine Tasse, vermutlich mit Kaffee, die er der Frau im Zentrum anbietet.

Das Thema des Bildes ist nicht nur die globale Verflechtung von Dienstleistungen und Waren, sondern die Bedingungen, unter denen diese produziert werden. Der Titel des Bildes, „Your Own Personal Slaves", weist schon darauf hin, dass die dargestellten Produkte - Smartphones, Kleidung, bestimmte Nahrungsmittel wie Schokolade, Shrimps und Kaffee, Öle für Kosmetika und Pornografie - vielfach unter ausbeuterischen Bedingungen hergestellt werden. Die Absicht des Bildes ist, Bewusstsein zu schaffen für die unmenschlichen Verhältnisse, unter denen Alltagsgegenstände erzeugt werden, und dabei die Verantwortung aufzuzeigen, die der westliche Konsumierende für die Aufrechterhaltung dieser Ausbeutungsverhältnisse hat, indem er diese Produkte kauft.

Auf der ikonografischen und ikonologischen Ebene ist es jedoch das Benennen der persönlichen Verantwortung, welches das Bild typisch für den skizzierten Trend des „bewussten Konsums“ macht. Mit dem Titel „Your Own Personal Slaves“ sowie zahlreichen bildhaften Elementen - dem Sitzen auf den Personen, den Blicken der Ausgebeuteten, der Anordnung des Bildes insgesamt - werden gesellschaftliche Produktionsverhältnisse personalisiert, was bedeutet: Sie werden nicht mehr als über die Instanz des Marktes vermittelt gedacht, sondern es wird eine persönliche Zuständigkeit, eine kausale Ursächlichkeit zwischen der Ausbeutung von Menschen und dem individuellen Akt des Konsums hergestellt. Indem etwa der dunkelhäutige Mann, dessen Haut nach dem Graben seltener Erden verfärbt ist, der Frau direkt ihr Smartphone reicht, werden gegenwärtige gesellschaftliche Verhältnisse als eine präkapitalistische Sklavenhaltergesellschaft dargestellt, in der nicht für den Markt, sondern direkt für eine Person produziert wird. Der Konsumierende wird demnach mit einem/einer Sklavenhalter/in gleichgesetzt, der/die für die Produktionsbedingungen unmittelbar und direkt verantwortlich ist, da schließlich er/sie dieses Smartphone gekauft hat.

Nochmals zugespitzt wird diese Aussage durch die Bildsymbolik des Che-Guevara-Shirts und der mit einer Blume geschmückten Flip-Flops der Frau im Zentrum. Beides lässt darauf deuten, dass die Person ihrer politischen Einstellung nach keinesfalls die Aus- 
Abbildung 1: Daniel Garcia: Your Own Personal Slaves.

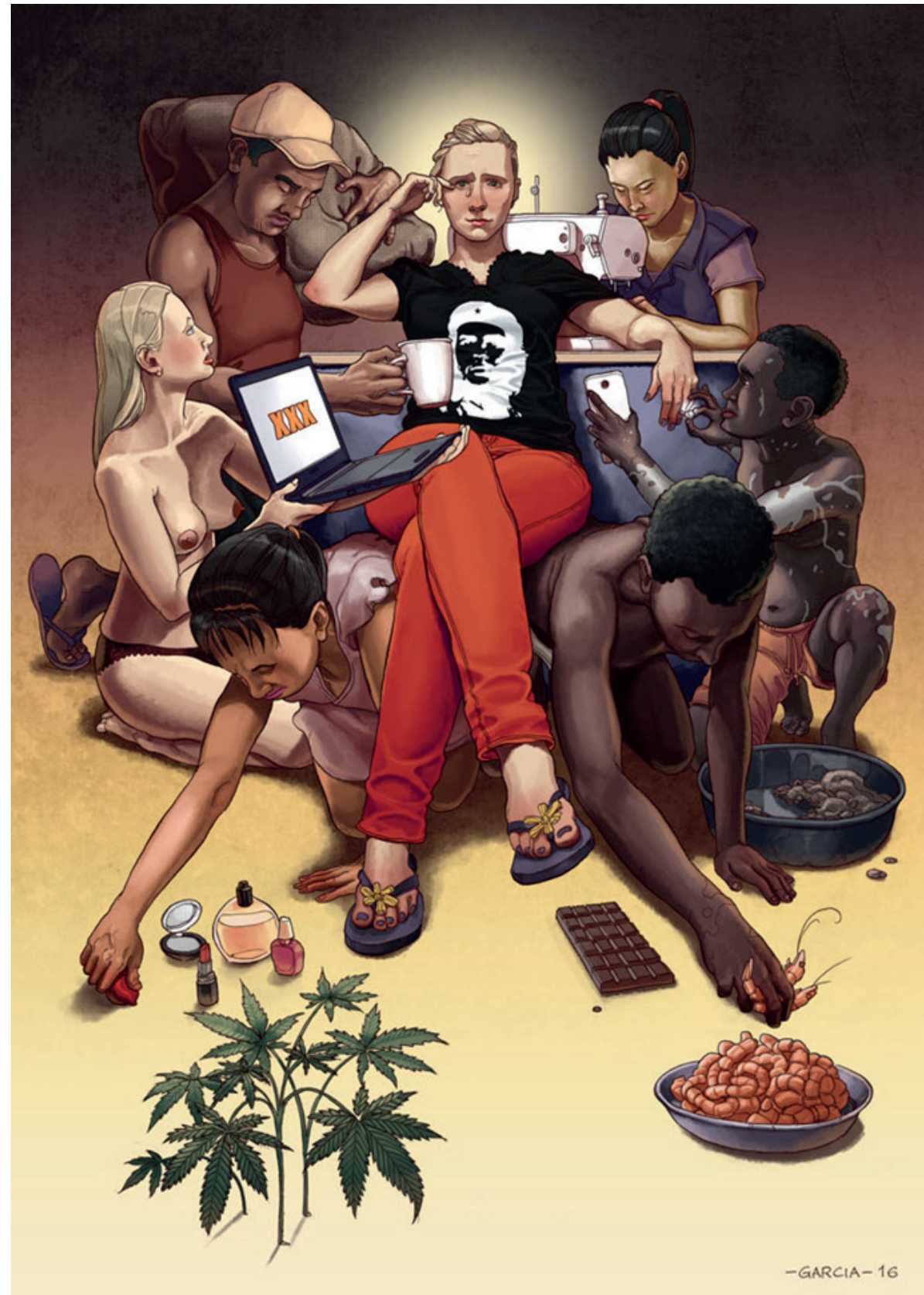

Quelle: http://www.danielgarciaart.com/your-own-personal-slaves/

beutung dieser Menschen gut heißen würde. Daher ist ihr Gesichtsausdruck auch ein ambivalenter: Sie scheint zwar um die Ausbeutung der Menschen durch ihren Lebensstil zu wissen, aber sie sieht weg - und konsumiert trotzdem. Sie scheint zwar zu wissen, dass sie mit ihrem Konsum für unmenschliche Arbeits- und Lebensverhältnisse verantwortlich ist -, aber sie schafft es nicht, aufzustehen, sondern bleibt auf den Rücken dieser Menschen sitzen. Die Aussage des Bildes ist an dieser Stelle, dass die westliche Konsumpraxis die Menschen im Grunde genommen gar nicht glücklich macht; dass sie jedoch ebenso wenig auf sie verzichten können. Daher schauen wie die Frau im Zentrum auch jene, die politisch gegen Ausbeutung sind, in ihrem Alltag weg und nehmen ausbeuterische Produktionsbedingungen hin, um weiterhin auf dem bestehenden Niveau konsumieren zu können, wenn auch mit einem weinenden Auge. In Anlehnung an ein Bonmot von Peter Sloterdijk könnte man daher formulieren: Der Konsumierende weiß genau, was er/sie tut, aber er/ sie tut es trotzdem (Zizek 1989: 25). Vor diesem Hintergrund müssen die Vehemenz und Endgültigkeit des 
Bildes, die einzelnen Konsumierenden zu Alleinverantwortlichen an den Zuständen auf anderen Kontinenten zu machen, interpretiert werden.

Dennoch bedingt die Abwesenheit aller anderen Faktoren und Instanzen, die zweifelsfrei ebenso als mitverantwortlich für Ausbeutung benannt werden können, eine dramatische Verkürzung und Verzerrung der existierenden gesellschaftlichen Machtverhältnisse. Die Frage, wer von den existierenden Verhältnissen neben den Konsumierenden eigentlich profitiert, wird durch das Bild ebenso wenig gestellt wie die Frage nach der historischen Entstehung von Ausbeutung und Strukturen, die diese Arbeitsverhältnisse erst ermöglichten. Die Komplexität dieser Problematik wird ersetzt durch einen einfachen Ursache-WirkungsZusammenhang, der bei Konsumierenden alle Macht und Verantwortung für sich selbst und den Planeten erkennt und bei den Ausgebeuteten völlige Abhängigkeit von und Angewiesenheit auf westliche Konsumierende. Gerade dieser Punkt ist es jedoch, der das Bild in Bezug auf die Frage nach der Entstehung der Subjektpositionen der abgehängten Konsumierenden und des moralisch höherwertigen Konsums so interessant macht.

\section{Abgehängte Konsumierende}

Wenn behauptet wird, dass der Konsumierende durch seine Kaufentscheidung die Macht hätte, Ausbeutungsverhältnisse $\mathrm{zu}$ beenden, so wird eine Kaufentscheidung in ein Disktinktionsmerkmal transformiert: So kann unterschieden werden zwischen dem bewussten oder moralischen Konsum jener, die mit ihrer Kaufentscheidung die Produktionsverhältnisse ihrer Produkte berücksichtigen und damit Ausbeutungsverhältnisse zu beenden versuchen; oder aber jenen, die diese nicht berücksichtigen und sich damit moralisch schuldig machen an dem Fortbestehen von Ausbeutung. Diese Unterscheidung zeigt sich nicht zuletzt gut in Produktwerbungen, in denen die Zielgruppe moralisch bewusster Konsumierender angesprochen wird; aber auch offensiver in Aussagen, in denen Konsumierende angegriffen werden, wenn sie Produkte kaufen, die den Standards der moralisch Bewussten nicht genügen, oder auch, wenn sie „zu viel“ konsumieren. Denn eine hegemoniale Aussage bewusster Konsumierender lautet, dass ein möglichst „minimalistischer Lebensstil“ glücklicher machen würde als exzessiver Konsum; Magazine wie der Stern geben Hinweise, „Wie man mit weniger Krempel ein glücklicheres Leben führen kann“
(Sellmair 2017); das ökologisch orientierte Magazin Utopia veröffentlichte einen Ratgeber mit „7 Tipps, die dir dabei helfen, weniger zu konsumieren“, wobei der letzte dieser Tipps war, überhaupt nichts Neues zu kaufen - aber wenn, dann „konsumiere ökologisch und ethisch produzierte Produkte“ (Jakob 2018, online).

In einem Beitrag des Magazins Sterns heißt es, das Deutungsangebot des minimalistischen Lebensstils sei eine „doppelte Verheißung: Man rettet - vielleicht die Erde und - ganz bestimmt - sich selbst. Wer sich bescheidet, [...] befreit sich aus den Zwängen von Schuften und Shoppen, Besitzen und Entrümpeln und kann sich so aufs Wesentliche konzentrieren - Beziehungen, Erlebnisse, Arbeit, die Freude macht. Er fastet nicht nur zwischen Aschermittwoch und Ostern - er verzichtet länger, umfassender, für ein besseres Leben. “ Sellmair 2017); Diesem Deutungsmuster liegt die Annahme zugrunde, dass es genau eine richtige Art des Konsums gibt, die zugleich das Individuum glücklich macht und durch welche der machtvolle westliche Konsumierende seiner globalen Verantwortung gerecht wird. Es wird kein kausaler Zusammenhang behauptet - der Konsumierende wird nicht glücklich, weil er etwas Gutes tut, sondern es sind zwei moralische Argumente, die auf denselben Punkt abzielen. Wer anders handelt, etwa maßlos billige Kleidung kauft, schadet daher sowohl sich selbst als auch allen anderen Menschen auf dem Planeten. Er handelt also objektiv unvernünftig und unmoralisch - eine feste Verbindung von allgemeiner Moral und ontologischer Vernunft, die in der Philosophie Kants besteht, liegt auch diesem Argument zugrunde.

Nicht zuletzt deshalb fällt Kritik an Konsumierenden, die solcherart handeln, hart aus. In einem typischen Aussagemuster führt die unverantwortliche Konsumpraktik zur Feststellung, diese Menschen seien aufgrund ihrer moralischen und intellektuellen Verkennung der Verhältnisse wohl abgehängt. Der Status des Abgehängtseins wird dann mit weiteren vermeintlichen oder tatsächlichen Charakteristiken in Verbindung gebracht, die für ein bestimmtes soziokulturelles Milieu typisch sind - und somit entsteht eine Aussage, in der auf den subalternen Status von Menschen „ganz legitim" hinuntergeschaut werden kann, da sich diese - nach scheinbar objektiven moralischen und vernünftigen Kriterien - ja falsch verhalten.

In einem Artikel für die Tageszeitung der Freitag fasst die Journalistin Katja Kullmann (2015) einige typische Aussagen zusammen. Sätze, die sie über Konsumierende aus sozial schwächeren Schichten hörte, 
waren die folgenden: „Im Ghetto wohnen - aber eine vollverspiegelte Karre mit Subwoofer-Sound fahren!“ Oder: „Keinen geraden Satz sprechen können, aber sich bei Primark dumm und dämlich shoppen! Guck dir das Girlie da drüben an, wie viele Kilo Billigklamotten hat die wohl zusammengeramscht?" In solchen Sichtweisen drückt sich nicht nur eine grundlegende Verachtung für den Lebensstil einer als minderwertig und abgehängt verstandenen sozialen Schicht aus, sondern es wird auch deutlich, dass diese für ihren Status keinerlei Mitgefühl verdienen. Vielmehr ist es ja gerade ihre Lebensweise, die ihnen zum Vorwurf gemacht wird, sie sind diesem Verständnis nach an ihrer Situation selbst schuld. Konsum wird in dieser Deutung nicht als ein soziologisches Symptom ungerechter sozialer Verhältnisse und Lebenschancen gesehen, als ein Akt der (zumindest angenommenen) Teilhabe an der Gesellschaft oder auch nur als eine schöne Stunde, in der man seinem tristen Alltag entflieht (und der demnach eigentlich das Problem wäre), sondern vielmehr als ein pathologisches Symptom von Unmoral und mangelnder Einsicht dieser Menschen.

Thorstein Veblen (1899) beschrieb schon vor über hundert Jahren die Funktion des Konsums, Distinktion herzustellen. Eine sozial höher gestellte Schicht wertet bestimmte Konsumpraktiken ihr unterlegener Schichten ab, um damit gleichzeitig die eigenen Praktiken als höherwertig zu positionieren und sich damit von der schlechter gestellten Schicht abzugrenzen. Dass die beiden Topoi der Sätze im vorhin genannten Beispiel - ein bestimmtes Auto fahren und maßloser Konsum von billiger Bekleidung - zwei zentrale Elemente jener Kritik des bewussten Konsums sind, ist insofern relevant, weil sich damit zeigt, dass auch die „neue“ Form des bewussten Konsums sich (bewusst oder nicht) dieses „alten“ Musters der Distinktion bedient. Anders formuliert: Hinter dem Vorwurf, nicht in nachhaltiger, ökologisch vertretbarer und bewusster Weise zu konsumieren, steckt in vielen Fällen wohl nicht selten ein Vorgang, der an Freuds Abwehrmechanismus der Rationalisierung erinnert. In ähnlicher Weise geht es wohl auch darum, unbewusst vorhandene Abscheu oder andere Gefühle, die hinter dem Wunsch nach Abgrenzung gegenüber einer bestimmten Person oder Gruppe stecken, so auszudrücken, dass sie sozial akzeptiert werden können, ohne ihren eigentlichen Charakter zu offenbaren.

In einem Artikel für die Frankfurter Allgemeine Zeitung gesteht sich das die Autorin Antonia Baum sogar selbst ein. Sie berichtet von einem Einkaufser- lebnis in der Textilkette Primark in folgender Art und Weise: „Bei einem T-Shirt von Marc Jacobs für sagen wir 180 Euro weiß man aber auch nicht und noch weniger, wer es wie gemacht hat. Aber man fühlt sich besser als bei Primark, wo viel Zeug auf dem Boden liegt, billiges Massenzeug, das man verachtet. Frauen mit ,Joyce'- und ,Chantal'-Tätowierungen laufen durch den Laden, man sieht das Maurerdekolleté von dicken Männern, die versuchen, sich Schuhe anzuziehen. Und vielleicht ist das Einkaufserlebnis beziehungsweise sein Image der Grund, der es einem so einfach macht, Primark zu verachten. Man hält die Menschen, die dort kaufen, für schlecht informiert, und denkt, auf der richtigen Seite zu sein, und liebt sich dafür. Man geht sich also ein Marc-Jacobs-Shirt kaufen und kauft das Gefühl, eine gute, bewusste Entscheidung getroffen zu haben (und: man gehört nicht zu denen, man gehört zu den anderen). Es ist der gute alte Wille zur Distinktion, der mit Kritik verwechselt wird, was mir aber gerade echt egal ist, weil ich mich setzen muss und nichts zum Setzen da ist." (Baum 2014, online).

Dieser Ausschnitt ist aufgrund der Offenheit der Autorin sehr bemerkenswert: Sie empfindet ein diffuses Gefühl des Unwohlseins im Geschäft, es ist zu eng, zu viel los, zu hektisch, Kleidungsstücke liegen am Boden und aus dem Lautsprecher dringt laute Technomusik. Sie verachtet die angebotenen Kleidungsstücke und vergleicht das „Einkaufserlebnis“ bei Primark mit einer „war zone“. Und obwohl es hier nahe liegen würde, weniger die anderen Menschen im Geschäft, ihr Aussehen und Verhalten zu fokussieren als beispielsweise sich die Frage zu stellen, wie die Anordnung des Geschäfts, die Einrichtung, die Werbung, die Preisgestaltung etc. mit jenem beobachteten Verhalten in Verbindung stehen könnte, macht die Autorin Ersteres: Sie richtet ihre Aufmerksamkeit auf die Primark-Konsumierenden (sie drängeln, shoppen $\mathrm{zu}$ viel, telefonieren zu laut, werfen Dinge auf den Boden, haben hässliche Tattoos) und macht diese für das negative „Einkaufserlebnis“ verantwortlich. Das Bemerkenswerte an diesem Artikel ist jedoch, dass der Autorin selbst die Funktion des Konsums, Disktinktion herzustellen, völlig bewusst ist. Ebenso weiß sie, dass das Argument des moralisch bewussten Konsums in Bezug auf Designerläden mit exquisiteren Marken wie Louis Vuitton oder Marc Jakobs ins Leere läuft, da diese Textilproduzenten genauso keine Auskunft über die Produktionsbedingungen geben. Sie weiß, dass man den Einkaufenden bei Primark daher eigentlich keinen Vorwurf machen kann, hier einzukaufen; aber dann 
schreibt sie, dass ihr das „aber gerade echt egal ist, weil ich mich setzen muss und nichts zum Setzen da ist".

Das ehrliche Bekenntnis in diesem Artikelausschnitt ist ein Indiz dafür, dass hinter Debatten um ein moralisch richtiges und vernünftiges Konsumverhalten manchmal wohl doch unbewusste Ressentiments stecken, die in rationalisierter Form auftreten. Die Autorin gibt zu, dass in ihrer Vorstellung ein „Einkaufserlebnis“ mit dem Besuch eines höherpreisigen Geschäfts einhergeht, in dem die gehobene Mittelschicht einkauft - denn dort zeigen Menschen die ihr gewohnten Formen sozialen Verhaltens, dort trifft sie auf Personen, die einen ähnlichen bürgerlichen Habitus mitbringen. Ebenso gibt sie zu, dass das gute Gefühl, das man mit dem Kauf des „richtigen“ Produktes erwirbt, vielmehr aus der Umgebung des Geschäfts entsteht als durch das Produkt selbst - das Geschäft zeigt, ob das Produkt richtig ist. Der Umkehrschluss lautet hier: In der falschen Umgebung gibt es nur falsche Produkte - Menschen, die dort einkaufen, müssen demnach die falsche Entscheidung treffen, sie handeln demzufolge wohl unvernünftig.

Dieses Deutungsmuster entspricht in einer wesentlichen Annahme dem vorhin analysierten Bild von Daniel Garcia: Es unterstellt unbegrenzte Handlungsmacht auf Seiten der Konsumierenden, die ihre Konsumentscheidung auf Basis völliger individueller Freiheit treffen können, weshalb jede Entscheidung eine bewusste ist (und eine falsche Entscheidung ist demnach eine bewusst falsche). Dem Hinweis, dass manche Menschen nur über begrenzte Mittel verfügen, begegnet diese Deutungsweise mit der Logik des Minimalismus: Konsum mache ja nicht glücklich - die Journalistin Antonia Baum will mit ihrer Beobachtung der Teenager, die bei Primark Unmengen von billigen Kleidungsstücken kaufen, eigentlich sagen: Niemand braucht so viel - sie wären besser dran, nicht so viel zu kaufen. Das Argument, Menschen aus schwächeren sozialen Schichten müssten eben in niedrigpreisige Geschäfte wie Primark gehen (wodurch ihr Verhalten bislang legitimiert war), gilt in einem Diskurs nicht mehr, der Konsumverweigerung als die beste Form des Konsums ansieht. Dass leistbare Business-Outfits, leistbare Smartphones und dergleichen hingegen vielen Menschen Zugang zu Jobs ermöglichen, die ansonsten Personen aus einer höheren sozialen Schicht vorbehalten wären, wird von diesem Diskurs ebenso radikal in Frage gestellt wie die „Demokratisierung des Konsums“ (Bänzinger 2015): Mit dieser Formulierung wird die zunehmende Verfügbarkeit besonderer Güter für Menschen aus ökonomisch schwächeren sozialen Schichten bezeichnet, was ein Effekt fallender Preise ist. Diese resultieren wiederum aus Effizienzsteigerungen einer globalisierten Wirtschaft. Somit sind mehr Konsumgüter für viele Menschen erschwinglich geworden und es ist nun in der westlichen Welt breiten Massen möglich, am sozialen Leben teilzuhaben, auch an jenen Orten und in jenen Praktiken, die ein bestimmtes Equipment voraussetzen. Die Demokratisierung des Konsums ist somit ein wesentlicher Mechanismus gegen die soziale Schließung von Orten der Gesellschaft. Das Deutungsmuster eines moralisch bewussten Konsums verkennt, dass bewusst produzierte Güter oder auf solchen basierende Dienstleistungen im Regelfall erheblich teurer und für Geringverdienende nicht einfach erschwinglich sind, sodass etwa die Teilhabe an musikalischen Aktivitäten oder das Ausüben bestimmter Sportarten schnell wieder Angehörigen höherer Schichten vorbehalten sein würden.

Das bisher Ausgeführte lässt sich folgendermaßen zusammenfassen: Im Bild von Garcia, aber auch in vielen Äußerungen zum Diskurs um Konsum finden sich relativ übereinstimmende Klassifikationsmerkmale, die eine Distinktion zwischen „ethischen“ und „vernünftigen“ und „unethischen“ und „schädlichen“ Konsumpraktiken generieren. Diese verfügen über einen Aufforderungscharakter und führen zu einer starken Individualisierung von gesellschaftlichen Konsumpraktiken im Diskurs. Dies bedeutet, dass diese von jedweder sozialen Dimension, von gesellschaftlichen Macht- und Produktionsverhältnissen abstrahiert werden, wodurch die Folgen und die Verantwortung für daraus entstehende Probleme dem Individuum zugeschrieben werden. Was folgt, ist nicht nur eine Stigmatisierung aller „nicht-bewussten“ Konsumpraktiken und Konsumierenden, sondern auch, dass jenen Konsumierenden keine Lösung angeboten wird, wie sie im Fall von geringen finanziellen Mitteln dennoch die für sie notwendigen Güter erwerben sollen. Das Bild Your Own Personal Slaves vermittelt diesen Konsumierenden Schuld, es macht ihnen ein schlechtes Gewissen, ohne sich ihrer Situation tatsächlich anzunehmen. In der weinenden Frau in der Mitte des Bildes verdichtet sich stark die Perspektive der bürgerlichen Mittelschicht auf das Thema Konsum: Die Person sieht traurig aus in der Fülle ihrer Güter. Sie könnte aber einfach aufstehen, sie scheint frei zu sein, niemand scheint sie zu zwingen, auf den anderen Personen sitzen zu bleiben - daher verdient sie unser Mitleid nicht.

Verschiedene zeitgenössische Beobachtung nehmen eine zunehmende Polarisierung der Gesellschaft wahr, die aus verschiedenen Mechanismen der Abwertung und Ausgrenzung resultiert. Die aus dem hier vorgestell- 
ten Material (das, wie erwähnt, keinesfalls den Anspruch erweckt, alle im Diskurs vorhandenen Sprecher/ innenpositionen wiederzugeben, sondern nur diese eine) ermittelten Deutungsmuster über den bewussten Konsum stützen diese These. Mit dem Beispiel sollen aber auch Erklärungshypothesen geliefert werden, wie distinktive Deutungsmuster insbesondere über die bildliche Ebene erzeugt werden. Im letzten Abschnitt sollen daher zwei Thesen über das Wechselspiel von Bild und Text vorgestellt werden.

\section{Thesen zur Interferenz von bildlichen und textlichen Elementen}

Eine erste These zum Verhältnis von bildlichen und textlichen Diskurselementen lautet: Grafiken und Bilder sind in einem deutlich höheren Maße in der Lage, Gefühle von Schuld, Schuldbewusstsein und Verantwortlichkeit zu produzieren und uns als schuldhafte Subjekte zu konstituieren. So wie in Althussers Theorie Moses im brennenden Dornbusch erkennt, dass "gerade er" gemeint ist, so erkennen wir in Garcias Bild im Blick der weinenden Frau auch, dass „genau wir“ gemeint sind, wenn wir das Leiden der uns unterworfenen Personen erkennen. Deren Blicke sollen uns suggerieren: Wir sind arm, weil ihr reich seid. Wir haben zu wenig, weil ihr zu viel habt. Das anonyme Leid der Produzent/innen, die wir ansonsten nicht hinter den Produkten erkennen würden, wird hier symbolisch personifiziert; durch die Perspektive dieses Bildes werden wir als die Verantwortlichen, die Schuldhaften, ebenso direkt angerufen. Auch wir sind keine anonyme Masse mehr, sondern es sind "genau wir“ gemeint. Texte können Fakten über die Situation im globalen Süden benennen und zu einer allgemeinen Veränderung der gesellschaftlichen Verhältnisse aufrufen; zu einer persönlichen Adressierung als schuldhafte Subjekte sind sie dagegen nicht in der Lage. Gleichzeitig ist die Perspektive des Bildes, die genau dies tut (aber dabei stark reduktionistisch agiert), nicht so einfach vom Tisch zu wischen, gerade weil wir durch Berichte wissen, dass hier objektiv Ausbeutungsverhältnisse existieren, von denen wir als Konsumierende profitieren.

Zweitens: Was ich mit der ausführlichen Darstellung der Deutungsmuster von „bewussten“ und „abgehängten“ Konsumpraktiken zeigen wollte sowie mit der Positionierung des „bewussten“ Konsums als vernünftig, ist jene ausschließende Tendenz, jener althergebrachte Wunsch zur Distinktion, die dem „bewussten Konsum“ historisch (etwa in der Bewegung der Eine-Welt-Läden) fremd ist und dessen ursprünglichen politischen Zielen zuwiderläuft. Damit wollte ich nicht behaupten, dass bewusster Konsum immer distinktiv ist und zwingend so sein muss, sondern vielmehr darstellen, dass in jüngster Zeit jene Operation im Diskurs nachzuweisen ist, die Foucault als „diskursive Verknappung“ bezeichnet hat. Diese Verknappung meint, dass eine gegenhegemoniale Aussage aus dem Diskurs von einer machtvollen Sprechergruppe aufgegriffen wird, sie aber um einen wesentlichen Teil reduziert wird und die verkürzte Aussage in einem anderen Kontext erscheint. Dadurch hat sich das ursprüngliche Postulat, generell und im Interesse der begrenzten Ressourcen des Planeten weniger zu konsumieren, auf eine bestimmte soziale Gruppe (nämlich ökonomisch schwächere Schichten) und eine bestimmte Konsumpraxis verengt, bedient sich dabei aber immer noch der progressiven Sprache der ursprünglichen Sprechergruppe. „Nachhaltiger Konsum“ klingt im gegenwärtigen Diskurs ungemein positiv, wird aber vielfach nicht gebraucht, um ebendiesen zu fordern, sondern einfach nur, um die Konsumpraxis schwächerer Schichten zu diffamieren.

Die Operation der diskursiven Verknappung ist mit Bildern noch einfacher durchzuführen. Die Wirkung eines Bildes, d. h. seine Rezeption und Interpretation, ist noch stärker von Begriffen und vom konkreten Kontext abhängig. Die Relation zwischen textlichen und bildhaften Elementen ist daher sehr viel loser als am Beispiel dieses Textes dargestellt. Hier erscheinen sie zusammengehörig und passend, weil das Bild und die herangezogenen Texte tatsächlich aus demselben Zeitraum stammen. Ob und wie dieses Bild aber in einigen Jahren interpretiert wird, ist nicht abzuschätzen, es könnte ähnlich sein, aber auch völlig anders, weil im Bild vieles vage bleibt, was dessen Charakter eben ausmacht. Die gemeinsame Wirkung von textlichen und bildhaften Elementen auf Subjektivierungsprozesse besteht daher darin, dass die textlichen Elemente eine Bandbreite an möglichen Interpretationen liefern, mit denen die Betrachtenden die unbestimmten Flecken des Bildes füllt, d. h. aktuelle Diskurse jenes Subjektivierungsangebots liefern, mit denen das Individuum über das Medium des Bildes diese Angebote entsprechend deutet und interpretiert.

\section{Fazit}

Zweierlei folgert aus den hier dargestellten Hinweisen über die Wirkung von Bildern für (politische) Subjektivierungsprozesse und das Deutungsmuster des bewusst Konsumierenden: 
Zum einen, dass die Ambivalenzen und Widersprüchlichkeiten der Diskurspositionen, die vor allem durch verkürzte Textbotschaften und Grafiken ausgeblendet und naturalisiert werden, dringend wieder zum Gegenstand des Diskurses gemacht werden sollten; umgekehrt sollten ethisch-moralische Standpunkte und Äußerungen gerade aus einer sich selbst als links einordnenden Diskursposition nicht eingenommen bzw. getätigt werden. Schuld ist keine politische Kategorie; umgekehrt muss - auch wenn dies in diesem Artikel vielleicht so verstanden wurde - nicht jede Praktik sozial schwächerer Milieus verteidigt werden, weil es sich dabei um eine politisch nahestehende Klientel handelt.

Tatsächlich sind zahlreiche Konsumpraktiken dieser Milieus weder aus Klima- und Umweltüberlegungen noch aufgrund der Ausbeutung von Tieren und Menschen rund um den Globus verteidigungswürdig. Es ist ein Gemeinplatz, dass eine gesicherte Existenz der Menschheit nur durch einen fundamentalen Wandel im Konsumverhalten möglich ist. Nicht hilfreich ist in diesem Zusammenhang eine Moralisierung ebenjenes nicht-nachhaltigen Konsums ebenso wie eine als volksnah verstandene politische Inszenierung einer „proletarischen Lebenskultur" bei Festen und Auftritten, wie sie von sozialdemokratischen und teils auch von VertreterInnen der grünen Parteien gerne unternommen wird, bei der zur Verteidigung von Schnitzeln, Autos und dergleichen ausgerückt wird. Was es benötigt, ist eine differenzierte gesellschaftliche Debatte, ohne Dramatisierung und Moralisierung, in der die Frage der tatsächlichen Kosten des Konsums akzentuiert wird und in der Möglichkeiten einer politischen Regulierung des Konsums erörtert werden.

Dies gilt auch - zweitens - in besonderem Maß für die politische Bildung. Oftmals wird die Frage des Konsums in der politischen Bildung z. B. als Verbraucher/ innenbildung verstanden und individualisiert betrachtet. Das Ideal lautet hier, dass mit dieser Bildung das Individuum zu einer mündigen Konsumentscheidung ermächtigt wird. Dies ist zwar zweifellos wichtig, jedoch kann auf diese Weise die Illusion entstehen, dass eine Veränderung einer gesellschaftlichen Konsumpraxis allein durch individuelle Konsumhandlungen unternommen werden kann, sodass hinter der Losung des „you can make a difference“ (was an sich schon zu bezweifeln ist) die Frage nach politischen Regulierungen oder Preispolitiken gar nicht mehr gestellt wird. Politische Bildung muss sich daher ebenfalls mit der gesellschaftlichen Dimension des Konsums und den politischen Antworten darauf befassen, ohne wiederum in die Diskursfalle einer moralischen Distinktion eines „notwendigen“ und „guten“ bzw. eines „überschießenden“ und „schädlichen“ Konsums zu tappen. Wichtiger wäre zu fragen - auch als Anstoß für weitere Forschung -, weshalb diese Zuschreibungen existieren, welche Strategien in diesem Diskurs verfolgt werden aber auch, wie Verhaltensänderungen breiter Teile der Bevölkerung erwirkt werden können, ohne dadurch soziale Ungleichheiten erneut zu zementieren.

\section{Literatur}

Althusser, L. (1971): Lenin and philosophy and other essays. New York: Monthly Review Press.

Bänziger, P-P. (2015): Von der Arbeits- zur Konsumgesellschaft? Kritik eines Leitmotivs der deutschsprachigen Zeitgeschichtsschreibung. Zeithistorische Forschungen, $12(1), 11-38$.

Bauman, Z. (2013): Consuming life. Hoboken: John Wiley \& Sons.

Baum, A. (2014): Ich bin grad Primark. Online: https://www. faz.net/aktuell/feuilleton/einkaufserlebnis-ich-bingrad-primark-13078450.html?printPagedArticle $=$ true $\# p$ ageIndex_o [Zugriff am 10. 8. 2020].

Butler, J. (2001): Psyche der Macht. Das Subjekt der Unterwerfung. Frankfurt a. M.: Suhrkamp.

Geimer, A./Burghardt, D. (2019): Die Mediatisierung von Subjektivierungsprozessen. In: Geimer A./Amling S./ Bosančić S. (Hg.): Subjekt und Subjektivierung. Wiesbaden: Springer, 235-257

Jakob, S. (2018): 7 Tipps, die dir dabei helfen, weniger zu konsumieren. Online: https://utopia.de/ratgeber/tipps-weniger-konsumieren-kaufen/ [Zugriff am 10. 8. 2020].

Johnston, J./Baumann, S. (2014): Foodies: Democracy and distinction in the gourmet foodscape. New York: Routledge.

Kauppert, M./Leser, I. (Hg.) (2014): Hillarys Hand: Zur politischen Ikonographie der Gegenwart. Bielefeld: Transcript Verlag.

Keller, R. (2011): Wissenssoziologische Diskursanalyse: Grundlegung eines Forschungsprogramms. Wiesbaden: Springer.

Kullmann, K. (2015): Plastikschrott in Jogginghosen. Online: https://www.freitag.de/autoren/katja-kullmann/plastikschrott-in-jogginghosen Zugriff am 10. 8. 2020 [Zugriff am 10. 8. 2020].

Panofsky, E. (1939): Studies in Iconology. Humanistic Themes in the Art of the Renaissance. New York: Routledge.

Sennett, R. (2007): The Culture of the New Capitalism. New Haven: Yale University Press.

Sellmair, N. (2017): Wie man mit weniger Krempel ein glücklicheres Leben führen kann. Online: https://www.stern.de/ wirtschaft/news/konsum--wie-man-mit-weniger-kremIIIIIIIIIIIIIIIIII 159 
pel-ein-gluecklicheres-leben-fuehren-kann-7411564. html [Zugriff am 10. 8. 2020].

Traue, B. (2013): Visuelle Diskursanalyse. Zeitschrift für Diskursforschung, 2 (1), 117-136.

Veblen, T. (1899): The Theory of the Leisure Class: An Economic Study of Institutions. London: MacMillan.

Žižek, S. (1989): The Sublime Object of Ideology. London: Verso.

\section{Bildnachweis}

Garcia, D. (2016): Your Own Personal Slaves. Online: http:// www.danielgarciaart.com/your-own-personal-slaves/ [Zugriff am 10. 8. 2020]. 